\title{
Desenvolvimento de Lasioderma serricorne (Fabricius, 1792) (Coleoptera: Anobiidae) em diferentes dietas e temperaturas
}

\author{
Lineuza Leite Moreira ${ }^{1 *}$ \\ Maria Aparecida Braga Caneppele ${ }^{1}$ \\ Sonia Maria Noemberg Lázzari ${ }^{2}$ \\ Alberto Dorval ${ }^{3}$ \\ Rosina Djunko Miyazaki ${ }^{4}$ \\ ${ }^{1}$ PPG em Agricultura Tropical, Faculdade de Agronomia e Medicina Veterinária \\ Universidade Federal de Mato Grosso, Cuiabá-MT, Brasil \\ ${ }^{2}$ Departamento de Zoologia da Universidade Federal do Paraná, Curitiba-PR, Brasil \\ ${ }^{3} \mathrm{PPG}$ em Ciências Florestais e Ambientais, Faculdade de Engenharia Florestal \\ Universidade Federal de Mato Grosso, Cuiabá-MT, Brasil \\ ${ }^{4}$ Instituto de Biociências, Departamento de Zoologia \\ Universidade Federal de Mato Grosso, Cuiabá-MT, Brasil \\ *Autor para correspondência \\ Avenida São Sebastião, n. 110, Cidade alta, CEP 78030-400, Cuiabá-MT, Brasil \\ lineuza@gmail.com
}

\section{Resumo}

Foram dispostos 10 casais de L. serricorne em 30g das dietas sorgo, milheto e camomila durante seis meses, para avaliar, diariamente, nas gerações subsequentes, o período de desenvolvimento (dias); viabilidade larval (\%); número de ínstares larvais e duração em dias de cada ínstar e do período pupal e longevidade dos adultos. Na temperatura de $20^{\circ} \mathrm{C}$, as dietas de milheto e sorgo não apresentaram diferença significativa, com duração média de 64,1 e 63,5 dias, respectivamente, para o estágio larval e de 24,1 e 24,5 dias para o estágio de pupa; mas, a $30^{\circ} \mathrm{C}$, a dieta de milheto mostrou-se mais favorável para o desenvolvimento de L. serricorne com duração média do ciclo biológico de 22,8 dias, enquanto que, a $25 \pm 2{ }^{\circ} \mathrm{C}$, milheto e camomila foram as dietas mais favoráveis, com duração média do ciclo biológico de 26,2 e 31,1 dias, respectivamente. A temperatura de $30^{\circ} \mathrm{C}$ e a dieta de milheto foram as mais favoráveis ao desenvolvimento de L. serricorne.

Unitermos: besourinho-do-fumo, biologia de insetos, Pennisetum sp., Sorghum sp.

\section{Abstract}

Development of Lasioderma serricorne (Fabricius, 1792) (Coleoptera: Anobiidae) in different diets at different temperatures. Ten couples of $L$. serricorne were placed on $30 \mathrm{~g}$ of diet, including sorghum, millet kernels and dried chamomile, kept for six months under different conditions. Several parameters were evaluated in the subsequent generations: development period (days); number of larval instars; larva viability (\%); duration of each instar and of the complete larval stage; and adult longevity. At $20^{\circ} \mathrm{C}$, the diets millet and sorghum did not show any significant differences, with mean durations for the larval stage of 64.1 and 63.5 days and for the 
pupa of 24.1 and 24.5 days, respectively. However, at $30^{\circ} \mathrm{C}$, the millet was more favorable for the development of $L$. serricorne than the other diets, with a biological cycle of 22.8 days. At room temperature $\left(25 \pm 2^{\circ} \mathrm{C}\right)$, millet and chamomile were more adequate for $L$. serricorne development, with a biological cycle duration between 26.2 and 31.1 days. In summary, the diet of millet at $30^{\circ} \mathrm{C}$ presented the best conditions for the development of $L$. serricorne.

Key words: cigarette beetle, insect biology, Pennisetum sp., Sorghum sp.

\section{Introdução}

A espécie Lasioderma serricorne (Fabricius, 1792) (Coleoptera: Anobiidae), considerada cosmopolita, causa considerável dano a uma grande variedade de produtos armazenados, incluindo oleaginosas, cereais, farinhas, folhas de fumo e frutas secas. Em tais produtos tanto as larvas quanto os adultos escavam galerias, danificandoos completamente (Powell, 1931; Stamatinis, 1935; USDA, 1972; Ryan, 1995).

A temperatura é um dos principais fatores ecológicos regulador das atividades dos insetos, podendo afetar a velocidade de desenvolvimento dos mesmos (Silveira Neto et al., 1976), sendo que cada espécie de inseto tem uma faixa de temperatura ideal, na qual os indivíduos atingem sua capacidade reprodutiva. $\mathrm{O}$ ciclo de vida de $L$. serricorne também é influenciado pela temperatura, umidade relativa e pela qualidade do alimento disponível (Powell, 1931; Ali et al., 1972).

A produção de grãos no estado de Mato Grosso é de extrema importância para a exportação brasileira. Grãos de importância econômica, como o sorgo, Sorghum spp., e o milheto Pennisetum spp. (Poaceae) podem ser atacados, exigindo a necessidade de monitoramentos com a finalidade de obter informações biológicas dessa espécie de inseto nesses hospedeiros.

Esta pesquisa teve por objetivo avaliar o desenvolvimento de L. serricorne em grãos de sorgo e milheto, mantidos em diferentes regimes de temperatura e umidade.

\section{Material e Métodos}

O experimento foi conduzido no Núcleo de Tecnologia em Armazenagem da FAMEV/UFMT. Aproximadamente 200 larvas de primeiro ínstar foram individualizadas em potes de plástico com tampa telada.
Essas foram alimentadas com $25 \mathrm{~g}$ de uma dieta que apresentava resultados de desenvolvimento satisfatório para esses indivíduos e diferenciada daquela que seria ofertada durante o experimento, evitando assim a preferência alimentar durante essa fase.

As larvas foram observadas diariamente até o estágio de pupa, as quais foram sexadas com base no dimorfismo sexual. Após esse procedimento, cada pupa foi colocada em um pote de plástico de $50 \mathrm{ml}$, etiquetado com a informação referente ao sexo do indivíduo. Após a emergência dos adultos formou-se 10 casais para acasalamento em diferentes dietas e temperaturas.

Para o estudo do desenvolvimento foram utilizadas $30 \mathrm{~g} /$ pote de três dietas diferentes: grãos de sorgo e milheto desinfestados em freezer e flores desidratadas de camomila. Os frascos contendo as respectivas dietas e indivíduos foram mantidos em três regimes diferentes de temperatura e umidade:

1. Temperatura ambiente: $25 \pm 2{ }^{\circ} \mathrm{C}$, umidade $70 \pm 5 \%$, monitoradas diariamente, no período de setembro a novembro de 2007;

2. Temperatura controlada de $20^{\circ} \mathrm{C}$ em estufa tipo B.O.D. (Biological Organisms Development) (Figura 2), umidade $50 \pm 5 \%$, fotofase de $12 \mathrm{~h}$, no período de setembro de 2007 a fevereiro de 2008;

3. Temperatura controlada de $30^{\circ} \mathrm{C}$ em estufa tipo B.O.D. (Biological Organisms Development), umidade $60 \pm 5 \%$, fotofase de $12 \mathrm{~h}$, no período de setembro a novembro de 2007 .

Após a obtenção da geração subsequente avaliaramse os seguintes parâmetros biológicos: o período de desenvolvimento (dias); viabilidade larval (\%); número de ínstares larvais e duração em dias de cada ínstar e do período pupal e longevidade dos adultos.

Considerou-se como melhor dieta aquela na qual o inseto realiza a mudança de ínstar em menor período de tempo. 
A comparação das médias foi realizada pelo teste de Scott - Knott a 5\% de probabilidade.

\section{Resultados e Discussão}

Em todos os tratamentos e nos três regimes de temperatura, registraram-se quatro ínstares larvais, com desenvolvimento das larvas nas três temperaturas testadas.

A duração média, em dias, do estágio larval de L. serricorne a $20^{\circ} \mathrm{C}$ para as dietas sorgo, milheto e camomila foi de 64,$1 ; 63,5$ e 66,1, respectivamente, sem diferença estatística significativa entre os tratamentos, exceto para larvas de $3^{\circ}$ ínstar que tiveram duração mais longa na dieta de camomila (Tabela 1).

Por se tratar de uma temperatura pouco favorável para o desenvolvimento de $L$. serricorne, a $20^{\circ} \mathrm{C}$ o estágio larval não parece ser influenciado pela alimentação, mas sim pela temperatura e umidade relativa. Estudos, como o de Niiho (1984), demonstraram efeitos desses fatores no desenvolvimento de $L$. serricorne, constatando que com umidade de $70 \%$ e valores médios de temperatura de $20 ; 22,5 ; 25 ; 27,5$ e $30^{\circ} \mathrm{C}$, o desenvolvimento desta espécie durou 217; 94; 81; 62 e 57 dias, respectivamente.
$\mathrm{Na}$ temperatura de $30^{\circ} \mathrm{C}$, para as dietas sorgo, milheto e camomila, a duração do estágio larval de L. serricorne, foi em média de 27,1;22,8 e 27,1 dias, respectivamente, mostrando que o inseto desenvolvese em todas as dietas estudadas. Para as larvas de $1^{\circ}$ e $4^{\circ}$ ínstar não se observou diferenças estatísticas significativas entre os substratos testados (Tabela 2). Contudo, para o $2^{\circ}$ e $3^{\circ}$ ínstares, a dieta de milheto, foi melhor do que a camomila e sorgo, acelerando a velocidade do desenvolvimento dos ínstares, refletindo também na duração total do estágio larval.

Essa diferença pode ser explicada pelas características da dieta, pois o teor de proteína do milheto é de $15,4 \%$ contra $11,2 \%$ do sorgo (Instituto Adolfo Lutz, 1985). O milheto tem melhor composição química, energética e maior porcentagem de proteína em relação ao sorgo, favorecendo o desenvolvimento dos insetos e de outros animais (Rostagno, 2000).

Trabalhos sobre a influência da dieta natural no desenvolvimento e taxa reprodutiva de L. serricorne constataram que tais parâmetros estão relacionados com os constituintes químicos da dieta larval. Ali et al. (1972) constataram que, em dieta com $26 \%$ de proteína e $50 \%$ de carboidrato, em temperatura de $30^{\circ} \mathrm{C}$ e UR

TABELA 1: Duração média dos ínstares de Lasioderma serricorne nas dietas de sorgo, milheto e camomila, a $20^{\circ} \mathrm{C}$ e fotofase de $12 \mathrm{~h}$, Cuiabá-MT, 2008.

\begin{tabular}{lcccc}
\hline \multirow{2}{*}{ Substrato } & \multicolumn{4}{c}{ Duração (dias) \pm desvio padrão } \\
\cline { 3 - 5 } \multicolumn{1}{c}{$1^{\circ}$ ínstar } & $2^{\circ}$ ínstar & $3^{\circ}$ ínstar & $4^{\circ}$ ínstar \\
Sorgo & $13,75 \pm 0,41 \mathrm{a}$ & $13,37 \pm 0,43 \mathrm{a}$ & $18,00 \pm 0,41 \mathrm{a}$ & $19,00 \pm 0,34 \mathrm{a}$ \\
Milheto & $13,50 \pm 0,41 \mathrm{a}$ & $12,75 \pm 0,43 \mathrm{a}$ & $18,12 \pm 0,41 \mathrm{a}$ & $19,12 \pm 0,34 \mathrm{a}$ \\
Camomila & $14,00 \pm 0,41 \mathrm{a}$ & $13,50 \pm 0,43 \mathrm{a}$ & $19,75 \pm 0,41 \mathrm{~b}$ & $18,87 \pm 0,34 \mathrm{a}$ \\
\hline CV $(\%)$ & 8,62 & 9,25 & 6,24 & 5,10 \\
\hline
\end{tabular}

Médias seguidas pela mesma letra na coluna não diferem estatisticamente pelo teste de Scott-Knott a 5\% de probabilidade.

TABELA 2: Duração média dos ínstares de Lasioderma serricorne, nas dietas de sorgo, milheto e camomila, em temperatura de $30^{\circ} \mathrm{C}$ e fotofase de $12 \mathrm{~h}$, Cuiabá-MT, 2007.

\begin{tabular}{lcccc}
\hline \multicolumn{1}{c}{ Substrato } & \multicolumn{4}{c}{ Duração (dias) \pm desvio padrão } \\
\cline { 3 - 5 } \multicolumn{1}{c}{$1^{\circ}$ ínstar } & $2^{\circ}$ ínstar & $3^{\circ}$ ínstar & $4^{\circ}$ ínstar \\
Sorgo & $2,81 \pm 0,09 \mathrm{a}$ & $6,25 \pm 0,29 \mathrm{c}$ & $5,56 \pm 0,24 \mathrm{~b}$ & $5,06 \pm 0,25 \mathrm{a}$ \\
Milheto & $2,81 \pm 0,09 \mathrm{a}$ & $3,18 \pm 0,29 \mathrm{a}$ & $3,56 \pm 0,24 \mathrm{a}$ & $5,31 \pm 0,25 \mathrm{a}$ \\
Camomila & $3,00 \pm 0,09 \mathrm{a}$ & $4,62 \pm 0,29 \mathrm{~b}$ & $6,25 \pm 0,24 \mathrm{~b}$ & $4,81 \pm 0,25 \mathrm{a}$ \\
\hline CV $(\%)$ & 13,60 & 25,28 & 19,49 & 20,15 \\
\hline
\end{tabular}

Médias seguidas pela mesma letra na coluna não diferem estatisticamente pelo teste de Scott-Knott a $5 \%$ de probabilidade. 
70 a $75 \%$, a duração do estágio larval foi de 25,20 ; 26,30; 31,69; 41,3 e 51,7 dias em sementes de Illicium verum (anis-estrelado); de Gossypium sp. (algodão); de Triticum aestivum (trigo); em Daucus carota (cenoura) e Beta vulgaris (beterraba), respectivamente.

A duração média do estágio larval (quatro ínstares) de $L$. serricorne foi de 31,$1 ; 26,1$ e 31,1 dias para as dietas de sorgo, milheto e camomila, respectivamente (Tabela 3).

Verificam-se diferenças estatísticas significativas entre as dietas na duração do período larval, mostrando que as dietas com melhor desempenho foram o milheto e a camomila. No caso do milheto, pode estar relacionado com a porcentagem de proteína em sua constituição (Rostagno, 2000), enquanto na dieta de camomila pode ser que em condições de ambiente natural essa dieta apresente algum componente químico que auxilie no desenvolvimento de $L$. serricorne.

Os resultados reforçam os obtidos por Ali et al. (1972), pois a dieta com maior concentração de proteína, e na faixa de temperatura e umidade relativa considerada favorável, apresentou desenvolvimento semelhante ao mencionado em sua pesquisa.

\section{Duração dos estágios de pré-pupa e pupa}

O período de pré-pupa caracteriza-se pela inatividade e falta de alimentação; a larva inicia a construção de um envoltório rígido constituído pelo material alimentar associado a suas secreções. $\mathrm{Na}$ temperatura de $20^{\circ} \mathrm{C}$, não houve diferença estatística na duração de pré-pupa em nenhuma das dietas, com médias de 10,6; 11,1 e 10,7 dias em sorgo, milheto e camomila, respectivamente.
A duração do estágio de pupa apresentou média de 24,$1 ; 24,5$ e 26,1 dias, nas dietas de sorgo, milheto e camomila, respectivamente. Variando de 22 a 26 dias para as dietas de sorgo e milheto e de 25 a 28 dias para a dieta de camomila. Estatisticamente, o estágio de pupa apresentou menor duração em sorgo e milheto em relação à camomila.

Para a temperatura de $30^{\circ} \mathrm{C}$, o estágio de pré-pupa não apresentou diferença estatística para nenhuma das dietas, com médias de 2,2 dias para o sorgo e para a camomila e 2,3 dias para o milheto.

O estágio de pupa apresentou duração média de 5,1; 5,6 e 6,2 dias para as dietas sorgo, milheto e camomila, respectivamente, com variação de 4 a 7 dias. Os dados obtidos são semelhantes aos obtidos por Samuel et al. (1984), que mostraram variações de 4 a 12 dias no período pupal. Estatisticamente, as dietas de sorgo e de milheto apresentaram melhor desempenho, a fase de pupa apresentou menor duração para ambas as dietas em relação à camomila.

Em ambiente com temperatura $25 \pm 2^{\circ} \mathrm{C}$, no estágio de pré-pupa, não se observou diferenças estatística entre as dietas testadas, com duração média de 2,7;2,6 e 2,6 dias para as dietas de sorgo, milheto e camomila, respectivamente. Esse fato pode ser atribuído à própria fase em que se encontra o inseto, pois o indivíduo passa por um intenso processo de metamorfose, ocorrendo os fenômenos de histólise e histogênese na transformação para pupa, portanto a dieta não se constitui em fator limitante no processo, mas sim a temperatura, que pode influenciar decisivamente na duração desse estágio de desenvolvimento. Durante o processo de metamorfose, as faixas de temperatura e de umidade relativa apresentaram-se dentro da faixa ótima para o desenvolvimento desta espécie.

TABELA 3: Duração média dos ínstares de Lasioderma serricorne nas dietas de sorgo, milheto e camomila, sob condições ambiente com temperatura de $25 \pm 2^{\circ} \mathrm{C}$. Cuiabá-MT, 2007.

\begin{tabular}{lcccc}
\multicolumn{1}{c}{ Substrato } & \multicolumn{4}{c}{ Duração (dias) \pm desvio padrão } \\
\cline { 2 - 5 } \multicolumn{1}{c}{} & $1^{\circ}$ ínstar & $2^{\circ}$ ínstar & $3^{\circ}$ ínstar & $4^{\circ}$ ínstar \\
Sorgo & $3,81 \pm 0,18 \mathrm{~b}$ & $5,00 \pm 0,19 \mathrm{~b}$ & $5,43 \pm 0,16 \mathrm{~b}$ & $8,25 \pm 0,29 \mathrm{~b}$ \\
Milheto & $2,81 \pm 0,18 \mathrm{a}$ & $3,50 \pm 0,19 \mathrm{a}$ & $4,37 \pm 0,16 \mathrm{a}$ & $6,62 \pm 0,29 \mathrm{a}$ \\
Camomila & $2,68 \pm 0,18 \mathrm{a}$ & $4,81 \pm 0,19 \mathrm{a}$ & $6,25 \pm 0,16 \mathrm{c}$ & $7,37 \pm 0,29 \mathrm{a}$ \\
\hline CV (\%) & 22,68 & 17,27 & 12,04 & 15,89 \\
\hline
\end{tabular}

Médias seguidas pela mesma letra na coluna não diferem estatisticamente pelo teste de Scott-Knott a 5\% de probabilidade. 
O estágio de pupa apresentou média de 6,0;6,3 e 7,4 dias para as dietas de sorgo, milheto e camomila, respectivamente. Variando de 5 a 7 dias para sorgo e milheto, que não apresentaram diferenças estatísticas significativas entre si e de 5 a 9 dias para camomila, que apresentou diferença estatística significativa quando comparada com as demais dietas testadas.

\section{Longevidade dos adultos}

A longevidade dos adultos não pode ser acompanhada na faixa de temperatura de $20^{\circ} \mathrm{C}$, pois o desenvolvimento da espécie estendeu seu ciclo, extrapolando o prazo previsto para a concretização da pesquisa.

Na temperatura de $30^{\circ} \mathrm{C}$ a longevidade dos adultos foi de 15 a 21 dias em sorgo e milheto e de 12 a 14 dias em camomila. Esses resultados estão de acordo com os valores obtidos por Howe (1957), ao explicar que diferenças na longevidade dos adultos estão relacionadas ao tipo de alimentação na fase larval, com a temperatura e a umidade relativa.

A duração do ciclo biológico, de larva a adulto, na temperatura de $30^{\circ} \mathrm{C}$, foi de 27,$1 ; 22,8$ e 27,1 dias para as dietas de sorgo, milheto e camomila, respectivamente.

Em ambiente com temperatura de $25 \pm 2^{\circ} \mathrm{C}$ a longevidade dos adultos foi de 17 a 21 dias para todas as dietas. Em ambiente com temperatura de $25 \pm 2^{\circ} \mathrm{C}$ o somatório da duração de todas as fases de desenvolvimento foi de 31,$2 ; 26,2$ e 31,1 dias para as dietas de sorgo, milheto e camomila, respectivamente.

A partir dos resultados foi possível concluir que:

a) para a temperatura de $20^{\circ} \mathrm{C}$ as dietas a base de sorgo e milheto foram as mais favoráveis ao desenvolvimento de $L$. serricorne; a $30^{\circ} \mathrm{C}$ foi o milheto e a $25 \pm 2^{\circ} \mathrm{C}$, foram milheto e camomila; b)independente da temperatura, a dieta de milheto foi a mais favorável ao desenvolvimento de $L$. serricorne;

c) a temperatura mais favorável ao desenvolvimento de L. serricorne, independente da dieta foi a de $30^{\circ} \mathrm{C}$;

d)a duração dos estágios de desenvolvimento foi inversamente proporcional às temperaturas testadas, independente da dieta.

\section{Referências}

Ali, A. D.; Donia A. R.; El-Sawaf S. K. 1972. The influence of natural food on the development and reproductive rate of Lasioderma serricorne Fab. (Coleoptera, Anobiidae). Anzeiger fur Schädlingskunde, 76 (1): 14-16.

Howe, R. W. 1957. A laboratory study of the cigarette beetle, Lasioderma serricorne (F.) (Col., Anobiidae) with a critical review of the literature on its biology. Bulletin of Entomological Research, 48: 9-56.

Instituto Adolfo Lutz. 1985. Normas analíticas do Instituto Adolfo Lutz. Instituto Adolfo Lutz, São Paulo, Brasil, 533pp.

Niiho, C. 1984. Ecological study of the tobacco beetle. Lasioderma serricorne (F.) II Growth of tobacco beetle fed on breadcrumbs. Japanese Journal of Applied Entomology and Zoology, 28: 209216.

Powell, T. E. 1931. An ecological study of the tobacco beetle, Lasioderma serricorne Fabr., with special references to its life history and control. Ecological Monographs, 1: 333-393.

Rostagno, H. S. 2000. Tabelas brasileiras para aves e suínos: composição de alimentos e exigências nutricionais. UFV, Viçosa, Brasil, 141pp.

Ryan, L. 1995. Post-harvest tobacco infestation control. Chapman \& Hall, London, UK, 155pp.

Samuel, R.; Prabhu, V. K. K.; Narayanan C. S. 1984. Influence of spice essential oil on the life history of Lasioderma serricorne (F.). Entomology, 9: 209-215.

Silveira Neto, S.; Nakano, O.; Barbin, D.; Villa-Nova, N. A. 1976. Manual de Ecologia dos Insetos. Ceres, São Paulo, Brasil, 419pp. Stamatinis, N. C. 1935. The enemies of tobacco in warehouses, Ephestia elutella $\mathrm{Hb}$. and Lasioderma serricorne Fab. The biology and measures for their control. Communications of the Tobacco Institute of Greece, 4: 1-65.

USDA. 1972. Stored tobacco insects-biology and control. U. S. Department of Agriculture, Handbook, 233: 531-537. 